\title{
PLANEAMIENTO ESTRATÉGICO Y PRESUPUESTO EN LAS MICRO Y PEQUEÑAS EMPRESAS TEXTILES DEL EMPORIO COMERCIAL DE GAMARRA
}

\author{
STRATEGIC PLANNING AND BUDGETING IN THE MICRO AND SMALL TEXTILE \\ ENTERPRISES OF GAMARRA COMMERCIAL EMPORIUM
}

HANS JHORDAn SiLvaToRralva Universidad del Pacífico Lima, Perú

ORCID: https://orcid.org/0000-0003-3018-0738

Correo electrónico: h.silvatorralva@up.edu.pe

\section{RESUMEN}

Objetivo: Determinar la relación entre el planeamiento estratégico y el presupuesto en las Micro y Pequeñas Empresas textiles del emporio comercial de Gamarra. Método: Investigación cuantitativa, experimental, transversal y correlacional. El tamaño de muestra estuvo conformado por 65 Micro y Pequeñas Empresas textiles. La técnica de recolección fue la encuesta y el instrumento fue el cuestionario. Para responder a las hipótesis y objetivos se usó la prueba de correlación de Spearman. Resultados: Las Micro y Pequeñas Empresas alcanzaron un nivel regular en planeamiento estratégico $(63,1 \%)$ y en sus dimensiones: Formulación de estrategias $(75,4 \%)$, implantación de estrategias $(60 \%)$ y evaluación de estrategias (67,7\%); asimismo, en el presupuesto (46,2\%). Además, se evidenció relación significativa directa entre la planificación estratégica $(p=0,001$, $R h o=0,472)$ y el presupuesto, al igual que, la dimensión implantación $(\mathrm{p}=0,001, \mathrm{Rho}=0,489)$ y la dimensión evaluación ( $p=0,02$, Rho=0,371); sin embargo, no se encontró relación con la dimensión formulación $(\mathrm{p}=0,180, \mathrm{Rho}=0,168)$. Conclusiones: La planificación estratégica se relaciona directamente y de manera significativa con el presupuesto de las Micro y Pequeñas Empresas. Es decir, mientras más adecuada sea la planificación, el presupuesto será más adecuado.

Palabras clave: Formulación; implantación; evaluación; planificación estratégica y presupuesto.

\begin{abstract}
Objective: Determine the relationship between strategic planning and budgeting in Micro and Small Textile Enterprises of Gamarra's commercial emporium. Method: Quantitative, experimental, cross - sectional and correlational research. The sample size consisted of 65 Micro and Small Textile Enterprises. The collection technique was the survey and the instrument was the questionnaire. Spearman's correlation test was used to respond to hypotheses and objectives. Results: Micro and Small Enterprises reached a regular level in strategic planning (63.1\%) and in its dimensions: Strategies formulation $(75.4 \%)$, strategies implementation $(60 \%)$ strategies evaluation (67.7\%); also in the budget (46.2\%). Besides, it evidence a significant direct relationship between the strategic planning $(p=0,001, R h o=0,472)$ and the budget, just like the implementation dimension $(p=0,001, R h o=0,489)$ and the evaluation dimensión $(p=0,02$, Rho $=0,371)$; however, it was not found the relation with the formulation dimension $(p=0,180$, Rho $=0,168)$. Conclusions: Strategic planning is directly and significantly related to the micro and small enterprises budget. It mean, the more appropriate the planning be, the more appropriate the budget will be.
\end{abstract}

Keywords: Formulation; implementation and evaluation; strategic planning; budget. 


\section{INTRODUCCIÓN}

Con el pasar de los años, la preocupación más latente de la dirección en las organizaciones es encontrar los mecanismos mediante los cuales se logre enfrentar con éxito la incertidumbre, situándolo en un contexto económico cada vez más cambiante y dinámico, resulta indispensable para la satisfacción de las expectativas de los accionistas, para mantener la competitividad estratégica en la empresa (Hitt, Ireland, y Hoskisson, 2008) y, por ende, para que se genere valor agregado a la sociedad (Kramer y Porter, 2011). Ante esta situación, se torna cada vez más crítico el hecho de que las empresas cuenten con recursos necesarios para predecir los efectos de los sucesos del futuro para que así la dirección tome decisiones fundamentadas que logren mitigar los riesgos en forma idónea (Palacios, 2016).

Dada la creciente interrelación de la empresa con su entorno y la complejidad que caracteriza a la gestión de las empresas en la actualidad, la actividad de planeación o planeamiento adquiere una mayor importancia dentro del proceso administrativo (Robbins y Courtler, 2014). Esta mayor relevancia se debe a que esta actividad marca el punto de referencia a través del cual se establece una metodología que ayuda a la organización a adaptar su estructura y su estrategia a entornos cambiantes y dinámicos, de tal manera que la selección de los cursos de acción se desarrolle sistemática y ordenadamente con miras al cumplimiento de las metas y los objetivos trazados (Rojas, 2008; Martinez y Milla, 1999).

Por consiguiente y en línea con lo mencionado, según Boronat (2004), la elaboración de presupuestos debiera ser una prolongación del trabajo previamente realizado durante la planificación estratégica, ya que el ejercicio presupuestal brinda a las empresas la posibilidad de asignar los recursos necesarios definiendo prioridades estratégicas para conseguir la rentabilidad deseada.

A nivel nacional, nueve de cada diez empresas son mypes (micro y pequeñas empresas) y su actividad empresarial representa el 21,6\% del PBI local (Instituto Nacional de Estadística e Informática [INEI], 2016). Una de las industrias más importantes es la correspondiente al sector textil - confecciones la cual representa el 1,9\% del PBI Nacional y el $10 \%$ de la producción manufacturera (Sociedad Nacional de Industrias [SIN], 2018). De hecho, el valor agregado bruto de la industria textil y de confecciones representa el 7,4\% del valor agregado bruto total de manufactura, alcanzando así los 6,5 billones de soles dentro de un total de 88,5 billones de soles (SNI, 2018).

Según cifras del Instituto Nacional de Estadística e Informática, a junio del 2019, el número de establecimientos comerciales ascendió a 2 millones 662 mil 940 unidades.
Asimismo, al cierre del segundo trimestre del 2019, se crearon 72 mil 365 empresas, dándose de baja 31 mil 165, experimentando una variación del 41 mil 200 empresas. Además, del total de empresas el $99,1 \%$ son micro y pequeñas empresas (INEI, 2019).

Muchas de esas empresas han surgido producto del emprendimiento de las personas, sin tener un conocimiento técnico o profesional sobre cómo gestionar, por lo que han enfrentado distintos problemas. Un entorno que agrupa a innumerables micro y pequeños empresarios en Lima es el emporio comercial de Gamarra, el cual está dedicado principalmente al sector textil. (Huayhua y Quincho, 2017). Según, el Directorio Central de Empresas y Establecimientos del INEI, al año 2017, se habían registrado 33 002 establecimientos que desarrollaban actividades económicas en el emporio comercial, y que permitía emplear alrededor de 80183 personas. Además, el 99,6\% son micro y pequeñas empresas. El $88,7 \%$ del total de microempresas se dedican a actividades de comercio al por menor, al por mayor y manufactura (Gamarra y las cifras económicas más relevantes del emporio comercial, 2019). Actualmente, uno de los aspectos de la estrategia empresarial que no se está dando la importancia adecuada y que está relacionado con el logro de los objetivos, en un contexto de riesgo, es la gestión mediante un sistema integral presupuestario (Navarro, 2018, p.16).

Hoy se sabe que las mypes de Gamarra juegan un rol importante en la medida que contribuyen a la generación de empleo, promueven el crecimiento económico y reducen el nivel de pobreza. De hecho, durante el 2016, Gamarra generó ventas totales por 6618 millones de soles a partir de las campañas de las principales temporadas comerciales como Fiestas Patrias, Navidad y Año Nuevo. En este sentido, las medianas y grandes empresas concentraron el $39,1 \%$ de la facturación, mientras que las mypes alcanzaron el $61,9 \%$ y sus ingresos ascendieron a 4030 millones de soles (Ninahuanca, 2017). Sin embargo, estas empresas enfrentan una serie de obstáculos y limitaciones que impiden su desarrollo en el largo plazo, entre estos factores se encuentran los problemas administrativos, operativos, estratégicos y externos (Avolio, Mesones, y Roca, 2008).

Los problemas administrativos se centran en la estructura organizacional y en la habilidad para obtener y desarrollar los recursos necesarios, e incluyen sendos temas relacionados con el personal, las finanzas empresariales y la gerencia del negocio. Por su parte, los problemas operativos están relacionados a tratar de repartir los recursos eficientemente, por lo que suelen ser más comunes en áreas como marketing, operaciones y logística. Los problemas estratégicos abarcan la habilidad de los pequeños empresarios para ajustar sus productos o servicios a la demanda externa. Finalmente, los problemas externos 
incluyen temas de infraestructura, corrupción, tecnología y baja demanda (Avolio et al., 2008).

En relación con lo afirmado sobre los factores administrativos, una adecuada administración financiera constituye uno de los principales retos, ya que la carencia de estos conocimientos unida al nivel de informalidad de muchas de sus operaciones ocasiona que no se cuente con el respaldo suficiente para sus operaciones financieras. La contabilidad es relevante solo para cumplir con las obligaciones tributarias, por lo que no es utilizada para tomar decisiones estratégicas. La manera de efectuar proyecciones es mental y mecánica, por ende, los cálculos son aproximados y sujetos a errores, que pueden llevar a sobre compra de inventarios o generar pérdidas innecesarias. Precisamente, los problemas descritos anteriormente conllevan a que el desarrollo empresarial de las mypes sea limitado, debido a factores como: la baja capacidad de gestión empresarial, bajo acceso y uso inadecuado de las tecnologías de la información, dificultad de acceso al financiamiento e informalidad (Avolio et al., 2008).

Este panorama señala que no todas las empresas logran tener éxito, a pesar de estar en un emporio comercial de considerables transacciones económicas (Seminario y Torres, 2018). Entonces, surgen interrogantes como las siguientes: ¿qué elementos están influyendo en la gestión empresarial o la gestión estratégica?, ¿será el manejo del presupuesto un factor que está asociado a este resultado?, ¿qué tanto influye ejerce el presupuesto sobre la gestión estratégica?, entre otras. De esta forma, para afrontar estos problemas, los empresarios deben tomar decisiones e implementar ciertas estrategias cortoplacistas que, generalmente, son reactivas y no planificadas. En ese sentido, un plan estratégico sería una herramienta necesaria para brindar una ruta u orientación al empresario, de modo tal que logre sus objetivos y metas planteadas, además de contribuir a que su organización sea más proactiva (D'Alessio, 2013).

Un plan estratégico permitiría al empresario conocer el entorno que lo rodea en relación a factores económicos, culturales, políticos, sociales, entre otros. Lo anterior, ejercería influencia sobre sus acciones y, a partir de ello, se podría construir una visión y una misión, además de plantear objetivos y estrategias para el desarrollo de su organización. Actualmente, uno de los aspectos de la estrategia empresarial que no se le viene dando la importancia adecuada y que está relacionado con el logro de los objetivos, en un contexto de riesgo, es la gestión de los objetivos mediante un sistema integral presupuestario (Navarro, 2018), situación que ocurre frecuentemente en las micro, pequeñas y medianas del sector privado.

Según lo descrito, se ha considerado los siguientes antecedentes a nivel internacional Maisaroh, PH y Hadi (2019) describieron los factores que influyen en la planificación del presupuesto en las escuelas primarias de la provincia de Yogyakarta (Indonesia) en una muestra de 284 escuelas. Concluyeron que existe efecto significativo entre el planeamiento y la planificación presupuestaria, además no existe influencia en la variable planificación presupuestaria por parte de la dimensión participación en el trabajo.

Por su parte, Hou, Ho, Chung y Wong (2016) realizaron un estudio con el objetivo de determinar las restricciones presupuestarias estrictas y su ausencia de planificación estratégica. Estos investigadores concluyeron que se han ajustado restricciones presupuestarias junto con la ausencia de planificación estratégica esto demostró que son factores influyentes en la externalización de los servicios de gestión de instalaciones. Además, explicaron que la mentalidad de los empresarios en Hong Kong deberá pasar de apoyar el negocio principal a generar valor agregado en actividades para así mejorar la rentabilidad puesto que, probablemente, las restricciones presupuestarias no desaparezcan.

Fadol, Barhem y Elbanna (2015) llegaron a la conclusión de que existe una relación positiva entre la falta de recursos y el desempeño organizacional por las respuestas recogidas en los hospitales privados y públicos de los Emiratos Árabes Unidos. De esta manera corroboraron que la amplitud de la planificación estratégica juega un papel clave y mediador en la relación mencionada. Además, Chaparro (2012) realizó un trabajo de investigación en Colombia con el objetivo de especificar estrategias adecuadas para la elaboración del plan como orientación y líneas de actuación que faciliten el posicionamiento de la empresa en el sector. Concluyó que, en el análisis externo, la empresa está expuesta a diferentes oportunidades y amenazas que pueden llevar a la industria a una situación de insostenibilidad.

A nivel nacional el estudio de Arce (2018) buscó evaluar cómo el planteamiento estratégico se relaciona en el presupuesto, y concluyó que existe una relación entre ambas variables con un coeficiente de 0,79 determinada por el uso de la correlación de Rho Spearman. A su vez, obtuvo un coeficiente de 0,801 con la dimensión fase de formulación, un 0,84 con la dimensión fase de aprobación, un 0,801 con la dimensión fase de ejecución y un 0,81 con la fase de evaluación. Su principal recomendación fue dar la debida importancia a todas las fases del presupuesto, ya que esto dará resultados eficientes de acuerdo con lo plasmado en el planteamiento estratégico.

Saavedra y Hurtado (2017) en su proyecto de investigación, plantean como principal objetivo determinar la eficacia del plan estratégico institucional en la ejecución presupuestal de la Municipalidad Distrital Sanagorán, 
Provincia Sánchez Carrión, departamento La Libertad, 2017, demostraron que existe influencia por parte de la eficacia del plan estratégico institucional sobre la ejecución presupuestal de la municipalidad distrital Sanagorán a través del coeficiente Rho de Spearman $(0,94)$, donde el $93,1 \%$ es la eficacia del plan estratégico en una escala regular, mientras un 4,2\% lo encuentra en un nivel deficiente. Asimismo, Fuentes (2017) en su estudio realizado en el Instituto Peruano del Deporte concluyó, que la variabilidad del plan estratégico institucional dependerá de un cumplimiento del 48,8\% de la ejecución del presupuesto público. Por su parte, Guerrero (2017) realizó un estudio en los Hospitales de la ciudad de Lima, donde concluye que existe una correlación de 0,341 de relación significativa entre el presupuesto por resultados y la planificación estratégica.

Desde la perspectiva de la justificación práctica, esta investigación validó si se sostiene la importancia del planeamiento estratégico a nivel de estas empresas con evidencia empírica. Lo anterior podría ser de gran utilidad para las Mype textiles de otros sectores que no lleven formalmente el diseño de un plan para los objetivos de largo plazo que garanticen su crecimiento y sostenibilidad en el tiempo. En el plano de la justificación teórica, la investigación permitió evaluar diversos aspectos teóricos relacionados al planeamiento estratégico como parte de la administración estratégica. Además de corroborar si este último elemento es relevante al momento de llevar a cabo el ejercicio del presupuesto en este tipo de empresas.

De acuerdo con lo antes mencionado se concretó el objetivo: Determinar la relación del planeamiento estratégico y el presupuesto en mypes textiles del emporio comercial de Gamarra en el año 2019. Formulándose para ello los siguientes objetivos específicos: determinar la relación de la fase de formulación de estrategias y el presupuesto, así como determinar la relación de la fase de implantación de estrategias y el presupuesto y finalmente determinar la relación de la fase de evaluación de estrategias y el presupuesto. Y finalmente se generan las siguientes hipótesis: Existe relación significativa del planeamiento estratégico y el presupuesto, asimismo, existe relación significativa de la fase de formulación de estrategias y el presupuesto, así como existe relación significativa de la fase de implantación de estrategias y el presupuesto, por último, existe relación significativa de la fase de evaluación de estrategias y el presupuesto.

\section{MATERIALES Y MÉTODOS}

Es una investigación de tipo no experimental, cuantitativo, transversal y correlacional. Se tuvo como muestra 65 establecimientos de micro y pequeñas empresas textiles del emporio comercial de Gamarra. Se realizó un muestreo no probabilístico y la selección fue por conveniencia. Para obtener los datos de la investigación se encuestaron directamente al empresario o representante legal de la empresa textil de emporio de Gamarra. El instrumento fue el cuestionario, el cual se dividió en dos secciones.

El primero fue el cuestionario de Planeamiento Estratégico, el cual tuvo 18 ítems. Divididos 3 componentes: formulación de estrategias (6 ítems), implantación de estrategias (6 ítems) y evaluación de estrategias (6 ítems). A cada ítem se le asignó un puntaje según la respuesta. El segundo cuestionario fue el de Presupuesto conformado por 13 ítems, dividido en 4 componentes: fase de previsión (4 ítems), fase de formulación (3 ítems), fase de aprobación y ejecución (2 ítems) y fase de evaluación (4 ítems).

Finalmente, se utilizó el programa estadístico SPSS (Startical Product and Service Solutions) versión 25 en español, se consideró el coeficiente de correlación de Spearman, se tomó en cuenta un nivel de significancia del 5\%.

\section{RESULTADOS}

Se incluyeron a 65 establecimientos de Micro y Pequeñas empresas textiles del emporio comercial de Gamarra.

Tabla 1

Características generales de los empresarios del emporio Comercial de Gamarra 2019

\begin{tabular}{lcc}
\hline \multicolumn{3}{c}{ Características generales } \\
\hline \multicolumn{1}{c}{ Edad } & $\mathbf{N}$ & $\%$ \\
$\leq 35$ años & 16 & $24,6 \%$ \\
36 a 45 años & 20 & $30,8 \%$ \\
46 a 55 años & 17 & $26,2 \%$ \\
$\geq 56$ años & 12 & $18,5 \%$ \\
Sexo & & \\
Masculino & 40 & $61,5 \%$ \\
Femenino & 25 & $38,5 \%$ \\
Años de actividad & & \\
$\leq 5$ 5años & 14 & $21,5 \%$ \\
6 a 15 años & 35 & $53,8 \%$ \\
16 a 25 años & 12 & $18,5 \%$ \\
$\geq 26$ años & 4 & $6,2 \%$ \\
Cargo & & \\
Pequeños empresarios & 22 & $33,8 \%$ \\
Microempresarios & 43 & $66,2 \%$ \\
Total & 65 & $100 \%$ \\
\hline
\end{tabular}

Fuente: Encuestas obtenidas de los empresarios del emporio Comercial de Gamarra 
En la tabla 1, se muestra que, del total de encuestados, el $66,2 \%$ correspondía al grupo de microempresarios; y el $33,8 \%$, al de empresarios. El promedio de edad fue de 43,5 años y el $38,5 \%$ estuvo conformado por mujeres. Asimismo, los encuestados manifestaron tener una antigüedad entre 6 a 15 años en el rubro textil (53,8\%).

La figura 1 muestra que el 15,4\% de las mype alcanzó un nivel inadecuado en planeamiento estratégico; el 63,1\%, un nivel regular; y el $21,5 \%$, un nivel adecuado con respecto a esta variable.
La figura 2 muestra que el 46,2\% de las mypes alcanzaron un nivel regular en el presupuesto, mientras que el 32,3\% alcanzaron un nivel adecuado y el 21,5\% lograron un nivel inadecuado con respecto de esta variable.

A partir de los datos mostrados en la tabla 2, se colige que existe relación significativa directa débil entre la planificación estratégica y el presupuesto $(\mathrm{p}=0,001 ; \mathrm{Rho}=0,472)$. Es decir, con un presupuesto adecuado, las empresas también lograrán un nivel adecuado en planificación estratégica. El $42,9 \%$ de los encuestados que percibieron como adecuado

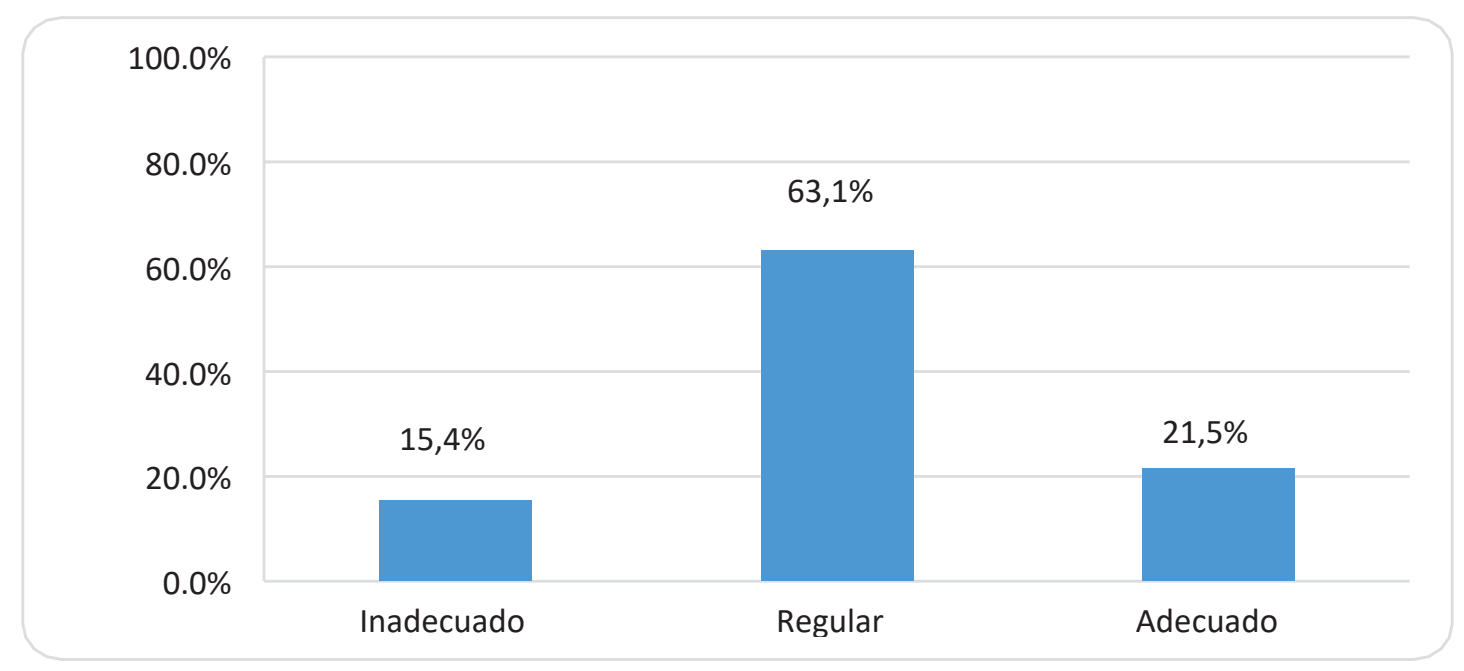

Figura 1. Nivel de planeamiento estratégico en mypes textiles del emporio comercial Gamarra 2019.

Fuente: Encuesta aplicada a los empresarios del emporio Comercial de Gamarra

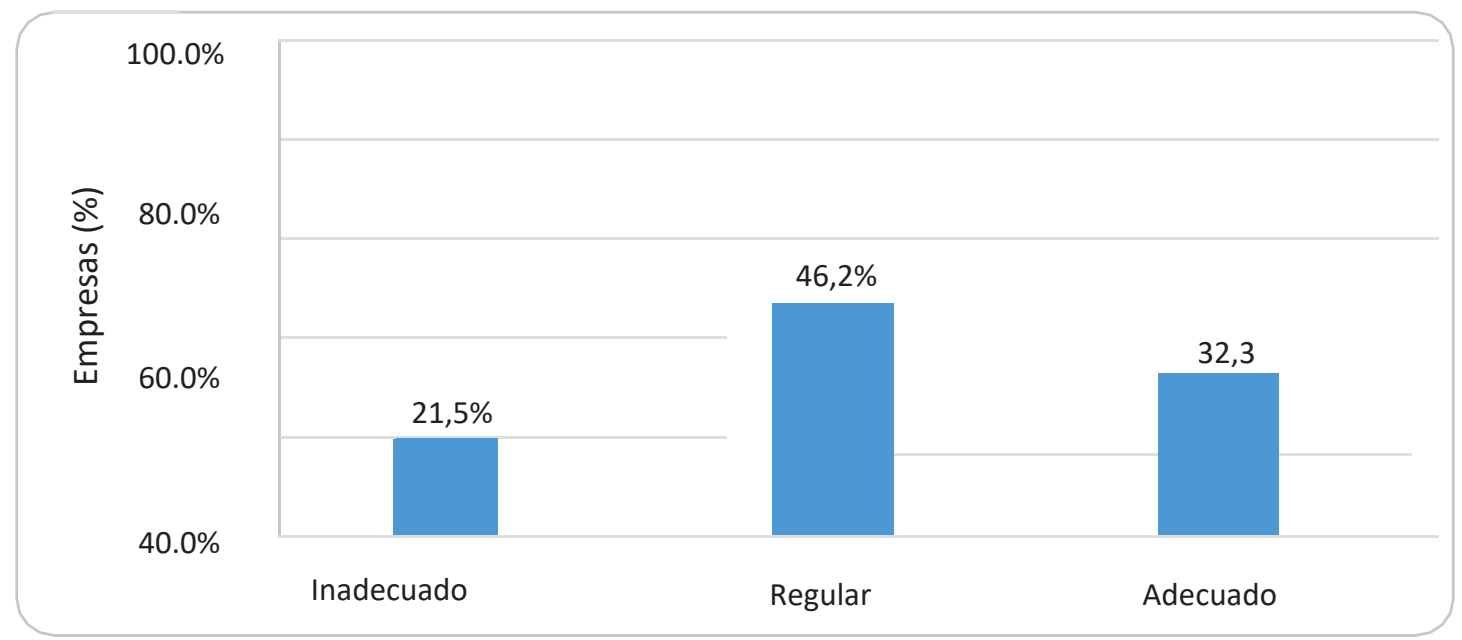

Figura 2. Nivel de presupuesto en las mypes textiles del emporio comercial de Gamarra 2019

Fuente: Encuestas obtenidas de los empresarios del emporio Comercial de Gamarra 
el presupuesto también logró un nivel adecuado en planificación estratégica. Este hecho se reflejó con lo que la gran mayoría afirmaba: a nivel interno, se identifican las fortalezas y debilidades que posee la empresa; se distribuyen los recursos adecuadamente, de tal forma que se lleva a cabo la estrategia formulada; en la empresa se ha declarado una misión y visión a largo plazo (4 años a más); se analizan las tendencias de los precios, costos, ventas, utilidad, etc., antes de realizar un presupuesto de forma efectiva, entre otros aspectos.

La tabla 3 muestra que existe relación significativa directa débil entre la fase de implantación estratégica $(\mathrm{p}=0,001$; Rho $=0,489)$, la fase de evaluación de estrategias $(\mathrm{p}=0,002$;
Rho=0,371) y el presupuesto. Esto se debe a que la gran mayoría de los representantes de los establecimientos de micro y pequeñas empresas textiles del emporio comercial de Gamarra se encontraban totalmente de acuerdo con que: a largo plazo (4 años a más) se establecen objetivos estratégicos; se revisan periódicamente los factores internos (fortalezas y debilidades) que pueden afectar las estrategias; se identifican las desviaciones favorables o desfavorables; y se prepara un informe con los resultados obtenidos.

En cambio, se evidenció que no existe relación significativa entra la fase formulación y el presupuesto ( $\mathrm{p}=0,180$; Rho=0,168); a pesar de que el $19 \%$ de mypes con presupuesto adecuado tuviese un nivel adecuado en la fase de

Tabla 2

Relación entre el planeamiento estratégico y el presupuesto en mypes textiles del emporio comercial de Gamarra 2019

\begin{tabular}{|c|c|c|c|c|c|c|c|c|}
\hline \multirow{3}{*}{ Planificación estratégica } & \multicolumn{6}{|c|}{ Presupuesto } & \multirow{3}{*}{$P^{*}$} & \multirow{3}{*}{ Rho } \\
\hline & \multicolumn{2}{|c|}{ Inadecuado } & \multicolumn{2}{|c|}{ Regular } & \multicolumn{2}{|c|}{ Adecuado } & & \\
\hline & $\mathbf{N}$ & $\%$ & $\mathbf{N}$ & $\%$ & $\mathbf{N}$ & $\%$ & & \\
\hline Inadecuado & 7 & $50,0 \%$ & 3 & $10,0 \%$ & 0 & $0,0 \%$ & & \\
\hline Regular & 5 & $35,7 \%$ & 24 & $80,0 \%$ & 12 & $57,1 \%$ & 0,001 & 0,472 \\
\hline Adecuado & 2 & $14,3 \%$ & 3 & $10,0 \%$ & 9 & $42,9 \%$ & & \\
\hline Total & 14 & $100 \%$ & 30 & $100 \%$ & 21 & $100 \%$ & & \\
\hline
\end{tabular}

Fuente: Encuesta aplicada a los 50 empresarios de Gamarra/*2rueba de correlación de Spearman.

Tabla 3

Relación entre las dimensiones de planificación estratégica y el presupuesto en mypes textiles del emporio comercial de Gamarra 2019

\begin{tabular}{|c|c|c|c|c|c|c|c|c|}
\hline \multirow{3}{*}{$\begin{array}{c}\text { Dimensiones de planificación } \\
\text { estratégica }\end{array}$} & \multicolumn{6}{|c|}{ Presupuesto } & \multirow{3}{*}{$\mathbf{P}^{*}$} & \multirow{3}{*}{ Rho } \\
\hline & \multicolumn{2}{|c|}{ Inadecuado } & \multicolumn{2}{|c|}{ Regular } & \multicolumn{2}{|c|}{ Adecuado } & & \\
\hline & $\mathbf{N}$ & $\%$ & $\mathbf{N}$ & $\%$ & $\mathbf{N}$ & $\%$ & & \\
\hline \multicolumn{9}{|l|}{ Formulación de estrategias } \\
\hline Inadecuado & 2 & $1, .3 \%$ & 5 & $16,7 \%$ & 0 & $0,0 \%$ & & \\
\hline Regular & 10 & $71,4 \%$ & 22 & $73,3 \%$ & 17 & $81,0 \%$ & 0,18 & 0,168 \\
\hline Adecuado & 2 & $14,3 \%$ & 3 & $10,0 \%$ & 4 & $19,0 \%$ & & \\
\hline \multicolumn{9}{|l|}{ Implantación de estrategias } \\
\hline Inadecuado & 5 & $35,7 \%$ & 4 & $13,3 \%$ & 0 & $0,0 \%$ & & \\
\hline Regular & 7 & $50,0 \%$ & 23 & $76,7 \%$ & 9 & $42,9 \%$ & 0,001 & 0,489 \\
\hline Adecuado & 2 & $14,3 \%$ & 3 & $10,0 \%$ & 12 & $57,1 \%$ & & \\
\hline \multicolumn{9}{|l|}{ Evaluación de estrategias } \\
\hline Inadecuado & 7 & $50,0 \%$ & 5 & $16,7 \%$ & 2 & $9,5 \%$ & & \\
\hline Regular & 6 & $42,9 \%$ & 24 & $80,0 \%$ & 14 & $66,7 \%$ & 0,002 & 0,371 \\
\hline Adecuado & 1 & $7,1 \%$ & 1 & $3,3 \%$ & 5 & $23,8 \%$ & & \\
\hline Total & 14 & $100 \%$ & 30 & $100 \%$ & 21 & $100 \%$ & & \\
\hline
\end{tabular}

Fuente: Encuesta aplicada a los 50 empresarios de Gamarra/* Prueba de correlación de Spearman. 
formulación de estrategias; y que el 14,3\% de las mypes con presupuesto inadecuado, un nivel inadecuado en esta fase.

\section{DISCUSIÓN}

Entre los hallazgos se evidenció que existe relación entre la planificación estratégica y el presupuesto $(\mathrm{p}=0,001$; Rho $=0,472)$, del tipo significativa $(\mathrm{p}=0,001)$ y directa $(\mathrm{Rho}=0,472)$. Resultados que son similares a lo reportado por Arce (2018), quien llegó a concluir la existencia de una relación significativa directa $(\mathrm{Rho}=0,791)$ entre las variables. Asimismo, fue para Saavedra y Hurtado (2017) quienes encontraron una correlación alta positiva entre la eficacia del plan estratégico institucional sobre la ejecución presupuestal. Por su parte, David (2013) menciona que la etapa de formulación se basa en identificar las oportunidades, amenazas, fortalezas y debilidades, además de crear la misión y visión. Los resultados arrojaron una relación directa entre fase Formulación de estrategias de la planificación estratégica y el presupuesto $(\mathrm{Rho}=0,168)$, sin embargo, no es significativa $(\mathrm{p}=0,180)$. Resultados que no concuerdan con lo reportado por Mogollón (2017) quien encontró que la formulación de la estrategia se relaciona de manera significativa con los Costos del Programa Presupuestal $(\mathrm{rho}=0,360, \mathrm{p}=0,000)$. Asimismo, fue para Arce (2018) quien halló que son estadísticamente significativa $(\mathrm{p}=0,000)$ se relaciona la fase formulación con el lineamiento estratégico. Respecto de la fase de Implantación estratégica se evidenció que existe relación directa entre la fase de implantación estratégica de la planificación estratégica y el presupuesto $(\mathrm{Rho}=0,489)$, siendo significativa $(\mathrm{p}=0,001)$. Es decir, a mayor la fase de implantación de la planeación estratégica es mayor el presupuesto. Resultados que concuerdan con lo reportado por Guerrero (2017) quien concluyó, la existencia de una relación significativa entre las variables estudiadas. Finalmente, sobre la fase de evaluación estratégica se evidenció que existe relación directa entre la fase de evaluación estratégica y el presupuesto $(\mathrm{rho}=0,371)$, siendo significativa $(\mathrm{p}=0,002)$. Es decir, a mayor la fase de evaluación de la planeación estratégica es mayor el presupuesto. Resultados que concuerdan con lo reportado por Arce (2018) quien concluyó que la relación entre el planeamiento estratégico y la fase evaluación fue significativa. Además, así fue para Guerrero (2017) quien encontró que el presupuesto por resultados se asocia a la evaluación de impacto. En conclusión, existe una asociación significativa directa entre la planificación estratégica y el presupuesto. Es decir, mientras más adecuada sea la planificación, el presupuesto será adecuado. También, se observó que casi la mitad de las mype con presupuesto adecuado tuvo una planificación estratégica adecuada y exactamente el $50 \%$ con presupuesto inadecuado, tuvo una planificación estratégica inadecuada. Ante lo hallado se ve la necesidad de profundizar en las variables estudiadas, ya que son de gran importancia por el aporte de nuevos conocimientos a los profesionales de administración, además de generar mayor evidencia sobre las variables en estudio y sus dimensiones. Asimismo, es importante buscar su asociación con otras variables como gestión administrativa y toma de decisiones. Se recomienda dar la debida importancia a las etapas del planteamiento estratégico para lograr resultados eficientes en el presupuesto, sobre todo en la formulación de estrategias, ya que son aquellas que identifican las oportunidades, amenazas, fortalezas y debilidades de una empresa.

\section{REFERENCIAS}

Arce, J. (2018). Planeamiento Estratégico y Presupuesto según el personal administrativo de la Unidad Ejecutora 002 de la Superintendencia Nacional de los Registros Públicos Sede Lima, 2016 (Tesis de Maestría). Universidad César Vallejo

Avolio, B., Mesones, A., y Roca, E. (2008). Factores que Limitan el Crecimiento de las Micro y Pequeñas Empresas en el Perú (MYPES). Lima: CENTRUM Católica.

Boronat, G. (2004). Presupuesto, beneficio y estrategia empresarial. Estrategia financiera, (210), 52-58.

Chaparro, C. (2012). Proceso de planeación estratégica aplicado en el contexto de la empresa boyacense. Revista In Vestigium Ire, 5(1), 7-16. Recuperado de: http://revistas.ustatunja.edu.co/index.php/ivestigium/ issue/view/43

D'Alessio, I. (2013). El proceso estratégico: Un enfoque de gerencia. México D.F.: Pearson Educación.

David, F. (2013). Conceptos de administración estratégica. México D.F.: Pearson Educación.

Fadol, Y., Barhem, B., y Elbanna, S. (2015). The mediating role of the extensiveness of strategic planning on the relationship between slack resources and organizational performance. Emerald Insight, 53(5), 1023-1044.

Fuentes, J. (2017). Ejecución del presupuesto público en el plan estratégico institucional del Instituto Peruano del Deporte - año 2016 (Tesis de Maestría). Universidad Cesar Vallejo.

Guerrero, L. (2017). Presupuesto por resultados y la planificación estratégica de la comunicación en los Hospitales de la Región Lima. 2016 (Tesis de Maestría). Universidad Cesar Vallejo. 
Hitt, M., Ireland, D., y Hoskisson, R. (2008). Administración estratégica: competitividad y globalización. México D.F.: Cengage Learning.

Hou, H., Ho, D., Chung, J., y Wong, K. (2016). Tight budget constraints and the absence of strategic planning - an exploratory analysis. Emerald Insight. 34(7), 380393. http://bit.ly/2lZ1nno

Huayhua, R., y Quincho, W. (2017). Análisis de las estrategias comerciales utilizadas por las PYMES del sector textil, del emporio comercial de Gamarra, de la línea de ropa para damas y caballeros con el fin de afrontar la competencia del mercado, y el impacto en su gestión comercial (tesis de licenciatura). Universidad Peruana de Ciencias Aplicadas (UPC).

Gamarra y las cifras económicas más relevantes del emporio comercial. (13 de marzo de 2019). El Comercio. Recuperado de https://elcomercio.pe/economia/peru/ gamarra-cifras-economicas-relevantes-emporio-comercial-fotos-victoria-george-forsyth-sunat-sen-noticia-616345-noticia/

Instituto Nacional de Estadística e Informática. (2016). Características de las Empresas del Emporio Comercial de Gamarra. Lima: INEI

Instituto Nacional de Estadística e Informática. (2017). Características de las empresas del Emporio Comercial de Gamarra, 2016. Lima, Perú. Recuperado de: http:// bit.ly/2krfXUi

Instituto Nacional de Estadística e Informática (2019). Demografía Empresarial en el Perú. II Trimestre 2019. Informe Técnico $N^{\circ} 3$. INEI. Lima, Perú. Recuperado de: http://bit.ly/2mn8MwY

Maisaroh, S., PH, S., y Hadi, S. (2019). The budget planning determinant factors at state primary schools in Yogyakarta province. International journal of instruction, 12(2), 354-368. http://bit.ly/2lzOboH

Martinez, D., y Milla, A. (1999). Cómo elaborar y controlar presupuestos anuales. Madrid: Esic S.A.
Mogollón, A. (2017). Planeamiento estratégico y costos del programa presupuestal control del cáncer del Instituto Nacional de Enfermedades Neoplásicas, Surquillo 2017 (Tesis de maestría). Universidad César Vallejo.

Navarro, H. (2018). El presupuesto como herramienta de gestión y sus efectos en la estrategia de las empresas agroindustriales de la Región Ica, 2014-2017 (Tesis de Maestría). Universidad de San Martin de Porres.

Ninahuanca, C. (13 de Julio de 2017). Gamarra generó ventas porS/ 6,618 millones en el 2016. El Peruano. Recuperado de http://www.elperuano.pe/ noticia-gamarra-genero-ventas-pors-6618-millones-el-2016-57555.aspx

Palacios, L. (2016). Dirección estratégica. Colombia: ECOE Ediciones.

Porter, M., y Kramer, M. (2011). Estrategia y sociedad: el vínculo entre ventaja competitiva y responsabilidad social corporativa. Harvard Business Review.

Robbins, S., y Courtler, M. (2014). Administración. México D.F.: Pearson Educación.

Rojas, M. (2008). Planeamiento y Presupuesto Estratégico. Lima: Entrelíneas S.R.L.

Saavedra, L., y Hurtado, H. (2017). Eficacia del plan estratégico institucional en la ejecución presupuestal de la Municipalidad Distrital Sanagorán, Provincia Sánchez Carrión, Departamento La Libertad 2017 (Tesis de maestría). Universidad Cesar Vallejo.

Seminario, K., y Torres, E. (2018). Diagnóstico del sector textil de las micro, pequeñas y medianas empresas (Mipymes) en el distrito de la Victoria - Lima metropolitana (Tesis de licenciatura). Universidad Peruana de Ciencias Aplicadas.

Sociedad Nacional de Industrias. (2018). Industria de textil y confecciones: Junio 2018. Lima: Autor. 